\title{
Computerbasierte Therapien: Eine Alternative in der modernen Psychotherapie? - Kontra
}

\author{
Computer-Based Therapies: A Supplement in Modern Psychotherapy? - Contra
}

Kontra

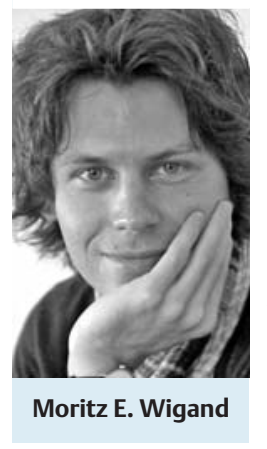

1997 schlug der Com-

puter Deep Blue den damals amtierenden Weltmeister Kasparow im Schach und im März 2016 besiegte das artificial intelligence-Programm AlphaGo den amtierenden Go-Weltmeister Sedol in diesem wohl komplexesten aller Brettspiele überhaupt [1]. Da scheint es nur folgerichtig, dass Computer inzwischen auch psychotherapeutische Aufgaben übernehmen, und es ist ein ehrenwertes Vorhaben, Menschen mit psychischen Störungen, die aus verschiedenen Gründen keinen ausreichenden $\mathrm{Zu}$ gang zum psychiatrisch/psychotherapeutischen Hilfesystem haben, einen Zugang zu entsprechenden Programmen zu verschaffen. Der Grundstein einer jeden Therapie ist es allerdings, den Patienten mit seiner individuellen Geschichte und seinem eigenen Erklärungsmodell wahrzunehmen und im besten Falle den therapeutischen Weg mit einem gemeinsamen Verständnis der Krankheitsmechanismen zu beginnen [2]. Dies wird nicht nur von Patienten gewünscht [3], sondern auch aus den Reihen der Psychiatrie angemahnt, so in Nancy C. Andreasens Kritik am mechanischen Abhaken von Diagnosekriterien, das einen „entmenschlichenden Einfluss“ auf die psychiatrische Praxis habe [4]. Mit der Einführung von Computerprogrammen in die Psychotherapie wird dieser Einfluss schmerzlich sichtbar. Beispielhaft sei das in Deutschland verfügbare Programm MoodGYM skizziert. Der Nutzer beantwortet Ratingskalen, wird durch psychoedukative Elemente geführt, lernt etwas über die „richtige und angemessene Denkweise“ und bekommt ein Feedback zu seinen Testantworten und seinem Erfolg. Begleitet wird er von holzschnittartigen Idealtypen mit sprechenden Namen; so werden „Miese- peter“ und „Anti“ einem infantil witzelnden „Nullproblemo“ gegenübergestellt, der wohl als Positivbeispiel oder Modell eines nicht depressiven Menschen dienen soll. Schon zu Beginn der „Therapie“ mit MoodGYM verwundert die Aussage, dass das Programm auf der Kognitiven Verhaltenstherapie und der Interpersonellen Therapie basiert. Eine interpersonelle Therapie, bei der es zu keinem interpersonellen Kontakt kommt, erscheint zumindest paradox. Solch ein Programm mit seinen vorgefertigten und erschreckend vereinfachten Pseudo-Antworten muss einem existenziell leidenden Menschen mehr als Hohn denn als Hilfe vorkommen, wird es doch weder der menschlichen Individualität des Patienten noch der Komplexität seiner Probleme gerecht. Über die Frustration der geringen Passung zwischen ihrer Situation und den Figuren berichten Betroffene:

When they [the characters] were relevant to me it was fine, you know, but when they weren't it was so frustrating.

(zitiert nach [5], S. 82)

Zahlreiche Studien (s. z.B. [6]) weisen auf den Zusammenhang zwischen sozialer Isolation, Einsamkeit und Depression hin. Insgesamt haben sich innerhalb einer Generation eine große Menge an täglichen Sozialkontakten, z.B. am Bankschalter oder beim Fahrkartenkauf, ohnehin schon durch den Einsatz von Computern und Automaten drastisch reduziert. Den psychisch kranken Menschen vor einen Bildschirm zu setzen scheint unangemessen, zumal wir wissen, dass im Gegenteil Programme, die ihn wieder unter Menschen bringen und sein Zugehörigkeitsgefühl zu einer Gruppe fördern, hilfreich sind [7]. Das Outcome einer Psychotherapie wird auf komplexe Art und Weise von der zur Anwendung kommenden Technik, der Therapeutenadhärenz (die nicht zu hoch und nicht zu niedrig sein sollte), der Kompetenz des Therapeuten, der therapeutischen Beziehung und Patientenvariablen beeinflusst [8]. In diesem komplexen Prozess sind eine Intuition, eine Flexibilität und ein Einfühlungsvermögen gefragt, die ein Computer nicht aufbringen kann. Die Psychotherapie ist, das zeigt sich spätestens in diesem Argument, ein komplexerer und auch menschlicherer Vorgang als jedes Brettspiel und kann nicht durch einen Computer ersetzt werden, wie auch folgende Aussage eines Betroffenen verdeutlicht:

If you're feeling like that, then a computer telling you something isn't going to make any difference. Whereas somebody seeing you and seeing the state that you're in can make a big difference ... slight physical things, just the words that she used ... you know, the verbal cuddle, which is what you need.

(zitiert nach [5], S.82)

In diesem Zusammenhang verwundert es nicht, dass die Unterstützung durch einen Therapeuten die Effektstärke internetbasierter kognitiver Verhaltenstherapie deutlich steigert [9], ein weiteres Indiz für die herausragende Stellung des Faktors Mensch.

Nun könnte gegen alle oben genannten Argumente eingewendet werden, dass computerbasierte Psychotherapie nicht dazu gedacht ist, den Therapeuten zu ersetzen, sondern nur als Add-on genutzt zu werden. Hierzu zwei Überlegungen: Wenn es tatsächlich nur als Add-on genutzt wird, stellt sich die Frage, welchen Zusatznutzen zu einem Therapeuten aus Fleisch und Blut der Computer bringen soll, denn nichts, das in den Programmen vermittelt wird, könnte nicht besser, menschlicher und authentischer vom Therapeuten selbst vermittelt werden. Wahrscheinlicher ist aber, dass diese Programme durchaus in Zukunft einen Teil der psychiatrisch/psychotherapeutischen Aufgaben übernehmen sollen. Ein Hinweis hierfür in Deutschland ist das Sponsoring durch eine große Krankenkasse, andererseits haben die Jahrhunderte der Industrialisierung und die vergangenen Jahrzehnte der Digitalisierung gezeigt, dass jeder Job, der durch einen Maschine ausgeführt werden kann, früher oder spä- 
ter auch durch eine Maschine ausgeführt wird. Schon gibt es eine „Roadmap“ zum breiten Einsatz computerbasierter Psychotherapie in den USA [10].

All diese inhaltlichen Gegenargumente könnten unter dem Gedanken der Zweck heiligt die Mittel über Bord geworfen werden, wenn durch computerbasierte Psychotherapien eine effektive Depressionsbehandlung erreicht würde. Alle Hoffnung aus bisherigen Studien (die größtenteils von den Entwicklern der Softwares selbst durchgeführt wurden) auf eine Wirksamkeit computerbasierter Psychotherapien wird jedoch durch eine neue methodisch hochwertige und pragmatische (d.h. realistische) Studie an $691 \mathrm{~Pa}$ tienten infrage gestellt [5]. Hier zeigte sich nicht nur im primären Outcome, Depressivität nach vier Monaten, keinerlei Effekt, sowie in den meisten sekundären Outcomes kein oder nur ein marginaler Effekt, sondern auch die Akzeptanz durch die Nutzer war denkbar gering: von 36 qualitativ befragten Nutzern waren nach der Therapie nur noch 9 positiv gegenüber dem Programm eingestellt, der Rest zeigte sich ambivalent oder negativ. Dass die Gesamtheit der Patienten eine computerbasierte Intervention nicht a priori ablehnte zeigt sich darin, dass vor der Intervention 65\% der Patienten der Intervention positiv gegenüberstanden. Weiterhin ist bemerkenswert, dass die Programme (MoodGYM und Beating the Blues ${ }^{\circledR}$ ) nicht einmal gegen eine fachpsychiatrische oder psychotherapeutische Behandlung, sondern lediglich als Add-on zur regulären hausärztlichen Versorgung getestet wurden.

Der Einsatz dieser (kaum wirksamen) Programme erscheint aus Sicht einer empathischen, personenzentrierten Psychiatrie bedenklich und letztlich wird sich (auch) hierin der Umgang der Psychiatrie mit den ihr anvertrauten Menschen im digitalen Zeitalter entscheiden.

\section{Literatur}

1 Borowiec S. AlphaGo seals 4-1 victory over Go grandmaster Lee Sedol. 15.3.2016. Im Internet: The Guardian; https://www.the guardian.com

2 Bhui K. Explanatory models for mental distress. Implications for clinical practice and research. Br J Psychiatr 2002; 181: 6 - 7

3 James CCAB, Carpenter KA, Peltzer K et al. Valuing psychiatric patients' stories. Belief in and use of the supernatural in the Jamaican psychiatric setting. Transcult Psychiatr 2014; 51: 247-263

4 Andreasen NC. DSM and the Death of Phenomenology in America. An Example of Unintended Consequences. Schizophr Bull 2006; 33: $108-112$

5 Littlewood E, Duarte A, Hewitt C et al. A randomised controlled trial of computerised cognitive behaviour therapy for the treatment of depression in primary care. The Randomised Evaluation of the Effectiveness and Acceptability of Computerised Therapy (REEACT) trial. Health Technol Assess 2015; 19: $1-174$

6 Barger SD, Messerli-Bürgy N, Barth J. Social relationship correlates of major depressive disorder and depressive symptoms in Swit- zerland: nationally representative cross sectional study. BMC Publ Health 2014; 14: $1-10$

7 Cruwys T, Alexander Haslam S, Dingle GA et al. Feeling connected again. Interventions that increase social identification reduce depression symptoms in community and clinical settings. J Affect Disord 2014; 159: 139 146

8 Barber JP. Toward a working through of some core conflicts in psychotherapy research. Psychother Res 2009; 19: 1-12

9 Spek V, Cuijpers PI, Nyklícek I et al. Internetbased cognitive behaviour therapy for symptoms of depression and anxiety. A meta-analysis. Psychol Med 2007; 37: 319

10 Cartreine JA, Ahern DK, Locke SE. A Roadmap to Computer-Based Psychotherapy in the United States. Harv Rev Psychiatry 2010; 18: 80-95

Sie haben eine eigene Meinung zu diesem Thema? Dann schreiben Sie uns an: psychiat-praxis@thieme.de!

\section{Korrespondenzadresse}

\section{Dr. med. Moritz E. Wigand}

Klinik für Psychiatrie und Psychotherapie II der Universität Ulm, Bezirkskrankenhaus Günzburg Ludwig-Heilmeyer-Straße 2

89312 Günzburg

moritz.wigand@bkh-guenzburg.de

Bibliografie

DOI http://dx.doi.org/

10.1055/s-0042-115318

Psychiat Prax 2017; 44: 11-12

(c) Georg Thieme Verlag KC

Stuttgart · New York

ISSN 0303-4259 